University of Wollongong

Research Online

Faculty of Engineering - Papers (Archive)

Faculty of Engineering and Information

Sciences

2005

\title{
Response of a SOI Microdosimeter to a /sup 238/PuBe Neutron Source
}

M. I. Reinhard

ANSTO, Australia

I. Cornelius

University of Wollongong, iwan@uow.edu.au

Dale A. Prokopovich

ANSTO, Australia, dap11@uow.edu.au

A. Wroe

University of Wollongong

Anatoly B. Rosenfeld

University of Wollongong, anatoly@uow.edu.au

See next page for additional authors

Follow this and additional works at: https://ro.uow.edu.au/engpapers

Part of the Engineering Commons

https://ro.uow.edu.au/engpapers/75

\section{Recommended Citation}

Reinhard, M. I.; Cornelius, I.; Prokopovich, Dale A.; Wroe, A.; Rosenfeld, Anatoly B.; Pisacane, V.; Zeigler, J. F.; Nelson, M. E.; Cucinotta, F.; Zaider, M.; and Dicello, J. F.: Response of a SOI Microdosimeter to a /sup 238/PuBe Neutron Source 2005.

https://ro.uow.edu.au/engpapers/75

Research Online is the open access institutional repository for the University of Wollongong. For further information contact the UOW Library: research-pubs@uow.edu.au 


\section{Authors}

M. I. Reinhard, I. Cornelius, Dale A. Prokopovich, A. Wroe, Anatoly B. Rosenfeld, V. Pisacane, J. F. Zeigler, M. E. Nelson, F. Cucinotta, M. Zaider, and J. F. Dicello 


\title{
Response of a SOI Microdosimeter to a ${ }^{238} \mathrm{PuBe}$ Neutron Source
}

\author{
M.I. Reinhard, Member, IEEE, I. Cornelius, D.A. Prokopovich, A. Wroe, A.B. Rosenfeld, Senior Member, IEEE, \\ V. Pisacane, J.F. Ziegler, Fellow, IEEE, M.E. Nelson, F. Cucinotta, M. Zaider and J.F. Dicello
}

\begin{abstract}
The response of a solid state microdosimeter based on semiconductor on insulator (SOI) technology to a ${ }^{238} \mathrm{PuBe}$ neutron source was investigated. Simulations of the device response using the GEANT4 Monte Carlo Toolkit are also presented. The microdosimetric spectra was measured for lineal energies above $1 \mathrm{keV} / \mu \mathrm{m}$. The possible application of the SOI microdosimeter for radiation protection applications in the form of a personnel microdosimeter is suggested.
\end{abstract}

\section{INTRODUCTION}

$\mathrm{T}$ HE knowledge of the radiobiological effectiveness of radiation (RBE), or the equivalent dose, is an important issue in radiation therapy and radiation protection.

Two different approaches have been suggested for estimation of RBE; the fluence based approach [1], and; the regional microdosimetry approach [2].

The fluence based approach requires the knowledge of all the particles contained in a mixed radiation field and the energy spectra. Using a weighting factor for each component, derived from radiobiological experiments, the equivalent dose can be determined. This approach involves particle identification instrumentation which is usually quite bulky.

The regional microdosimetry approach is based on the measurement of energy deposition events on the cellular level from secondary charged particles originating from the exposure of tissue to a mixed radiation field. The RBE in this case is given by $\int Q(y) y^{2} f(y) d \log y$, where $Q(y)$ is a quality factor obtained from radiobiological experiments and $f(y)$ is the spectrum of lineal energies $y$ (with units of $\mathrm{keV} / \mu \mathrm{m}$ ) measured by the microdosimeter.

The most commonly employed instrumentation type for measurement of the microdosimetric spectra are gas proportional counters [2]. Such instruments are bulky, demand a tissue equivalent (TE) gas supply, and high voltage operation which that make such instruments unsuited for use in personal radiation dosimetry.

Manuscript received November 10, 2005. This work supported by the National Space Biomedical Research Institute through NASA NCC 9-58.

M. Reinhard and D. Prokopovich are with the Australian Nuclear Science and Technology Organisation, Australia.

I. Cornelius, A Wroe and A. Rosenfeld are with the University of Wollongong, Australia.

V. Pisacane, J.F. Ziegler and M.E. Nelson are with the United States Naval Academy, Annapolis, MD 21402 USA.

F. Cucinotta is with the NASA Johnson Space Centre, Houston, TX 77058, USA.

M. Zaider is with the Memorial Sloan Kettering Cancer Care Centre, New York, NY 10021, USA.

J.F. Dicello is with The Johns Hopkins University, School of Medicine, Baltimore, MD 21231, USA.
Over several years the Centre of Medical Radiation Physics (CMRP) at the University of Wollongong has directed considerable effort towards the development of silicon microdosimeters based on silicon on insulator (SOI) technology. These devices consist of an array of $p-n$ junctions with sensitive volumes similar in size to biological cells. Investigation of the charge collection in the SOI microdosimeters and their application in radiotherapy can be found in $[3,4]$ and referenced papers contained within.

The purpose of this current work was to model and obtain experimental measurements using the SOI microdosimeter of the microdosimetric spectra of a mixed gamma-neutron field. The final aim of this research is the creation of a portable personal biological dosimeter based on the regional microdosimetric approach for use in mixed radiation fields.

\section{Materials AND MethodS}

\section{A. SOI Microdosimeter}

The SOI microdosimeter device is based on an array of $p-n$ junctions, (or cells), each with a planar area of $10 \times 10 \mu \mathrm{m}^{2}$ and a thickness of the sensitive volume of either $2 \mu \mathrm{m}, 5 \mu \mathrm{m}$ or 10 $\mu \mathrm{m}$. Figure 1 shows the topology of the SOI microdosimeter.

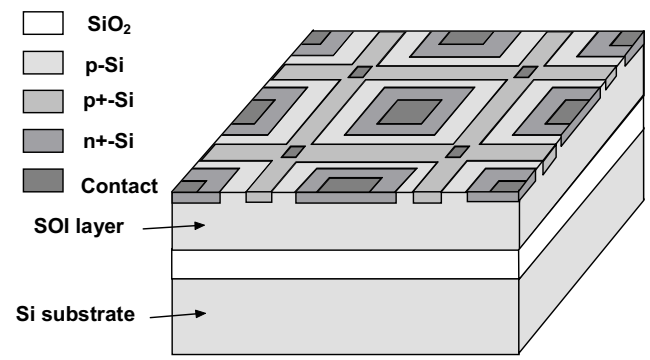

Fig. 1. Topology of the SOI microdosimeter.

The advantage of the SOI microdosimeter over TE gas chambers for microdosimetric measurements is the small size and weight, and the low voltage of operation $(5-10 \mathrm{~V})$. The charge collection in a single cell of the SOI microdoismeter and the chord length distribution for various sensitive volumes (SV) was studied previously [3]. It was demonstrated that such SOI microdosimeters can be used for lineal energies above $0.6 \mathrm{keV} / \mu \mathrm{m}(\mathrm{Si})$. The response in silicon measured over a wide range of energies for protons, alpha particles and 
electrons, typical for hadron radiation therapy, can be converted to an equivalent tissue response by scaling the sensitive silicon volumes with a coefficient of value 0.62 [5].

\section{B. Experimental Setup}

In the current work we investigated the response of the SOI microdosimeter in a mixed gamma/neutron field produced from a ${ }^{238} \mathrm{PuBe}$ radioisotope neutron source. The source was located within a general purpose neutron irradiation facility of floor area $7.5 \mathrm{~m}^{2}$. Walls $400 \mathrm{~mm}$ thick and $1.75 \mathrm{~m}$ high of borated paraffin and concrete surrounded the facility. The source activity was $325 \mathrm{GBq}$ with an estimated neutron emission rate of $2 \times 10^{7}$ neutrons in $4 \pi$. The average energy of fast neutrons was approximately $4 \mathrm{MeV}$ with a maximum energy of about $10 \mathrm{MeV}$. The dominant gamma component was a $4.4 \mathrm{MeV}$ emission associated with the decay of the bound excited state of ${ }^{12} \mathrm{C}^{*}$ produced in the $\mathrm{Be}(\alpha, \mathrm{n})$ reaction within the ${ }^{238} \mathrm{PuBe}$ source. A $5 \mu \mathrm{m}$ thick SOI microdosimeter was mounted at a distance of approximately $2.6 \mathrm{~cm}$ from the source.

The response of a $300 \mu \mathrm{m}$ thick silicon detector with $\mathrm{p}^{+} \mathrm{nn}^{+}$ structure was also measured at the same point. The purpose of the thick silicon detector measurements was to assist in the validation of GEANT4 based simulations of the experiment. Comprehensive studies of the response of thick silicon detectors to neutron fields for neutron spectroscopy purposes have been described by others $[6,7]$.

The neutron flux at the point of the SOI microdosimeter and thick silicon detector was $\sim 2.4 \times 10^{5} \mathrm{n} \mathrm{cm}^{-2} \mathrm{~s}^{-1}$ which corresponds to a dose rate of approximately $10 \mu \mathrm{Gy}^{-1}$. For neutron conversion polyethylene converters of various thickness were placed above the SOI microdosimeter and thick silicon detector. The converters had thickness of $0.1 \mathrm{~mm}$ and $0.5 \mathrm{~mm}$. The maximum thickness of $0.5 \mathrm{~mm}$ was sufficient for recoil proton charge particle equilibrium for neutrons up to $6 \mathrm{MeV}$ in energy which covers the majority of the $\mathrm{PuBe}$ neutron spectrum. Measurements without the converter layer were also performed to determine the contribution of silicon recoils and inelastic reaction products generated in the silicon from direct interactions of the neutrons with the silicon nuclei.

The data acquisition system consisted of a charge sensitive preamplifer (AMPTEK A250), shaping amplifier and multiple channel analyser (MCA). The system was calibrated in terms of energy (keV) using a spectroscopic grade ${ }^{241} \mathrm{Am}$ alpha particle source and a thick $(300 \mu \mathrm{m})$ planar detector with a capacitance similar to that of the SOI microdosimeter. The MCA spectra were acquired for both the SOI microdosimeter and silicon planar detector. The MCA spectra of the SOI device were later converted to a microdosimetric representation using a chord length of $5 \mu \mathrm{m}$.

\section{Simulations}

Simulations of the response of both the thick silicon detector and the SOI microdosimeter to the mixed neutron gamma field were carried out. The simulations were written in $\mathrm{C}++$ using classes taken and modified from the base classes of the GEANT4 Monte Carlo toolkit. Classes were used to model the geometry, primary beams, physics of interaction, and the collection of each particle history for the purpose of analysis. The geometry used in the simulation for the SOI microdosimeter was built from previously successful models used in the evaluation of this technology in fast neutron therapy [8].

The simulated neutron spectra for the ${ }^{238} \mathrm{PuBe}$ radioisotope source was obtained from an IAEA technical report [9]. Experimental verification of the neutron spectra was performed using a series of bonner spheres. The gamma spectra used for simulations was obtained from experimental measurement using a $\mathrm{NaI}(\mathrm{Tl})$ probe.

\section{RESULTS}

\section{A. Planar Silicon Detector Response}

Figure 2 shows the response of the bare (no polyethylene converter) thick silicon detector to the mixed radiation field. The simulated device response is also shown.

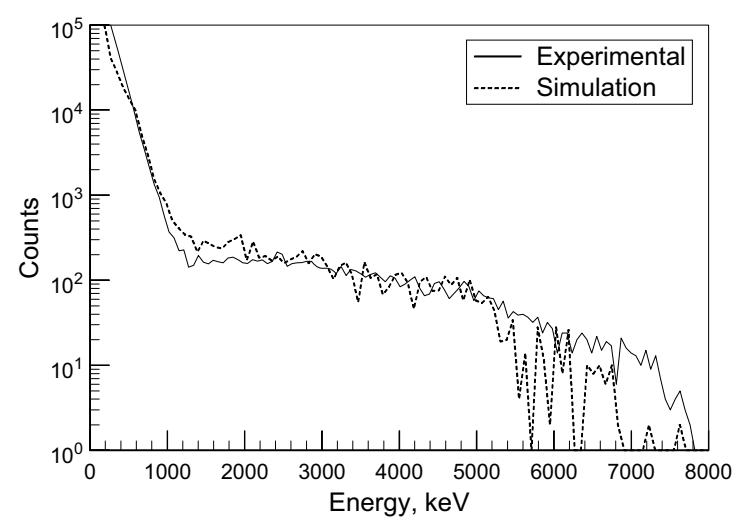

Fig. 2. Response of the bare (no polyethylene converter) thick silicon detector to the mixed radiation field. Spectra obtained from both experimental measurement and GEANT4 based simulations are shown.

The low energy exponential decay displayed in both spectra is a result of two contributing factors. The first was energy deposition by Compton events associated with the gamma component of the field. This component was almost independent of the presence of the converter, which has a thickness less than the range of secondary electrons, and is associated with the direct interaction of the photons within the $300 \mu \mathrm{m}$ of silicon. The second was elastically scattered silicon recoils from the $\mathrm{Si}(\mathrm{n}, \mathrm{n})$ reaction channel. The maximum energy transferred to a silicon nucleus by a $10 \mathrm{MeV}$ neutron is $1.32 \mathrm{MeV}$. An associated edge in the spectra at $\sim 1.3 \mathrm{MeV}$ is visible.

The events with energies above $1.3 \mathrm{MeV}$ are due to ionizing products released in inelastic reactions between the 
neutrons and the silicon nuclei of the detector bulk eg. (n,n'), $(n, p),(n, \alpha)$ and $(n, 2 p)$. These events in silicon should be taken into account in analyzing the microdosimetric spectra from the point of view that such high LET events generated in real tissue can be different than in silicon [8].

Close agreement between the experimental and simulated spectra was observed. Above $6 \mathrm{MeV}$ the reduced correlation was attributed to poor event statistics.

Figure 3 shows the experimentally measured and simulated spectra for the case of a $0.1 \mathrm{~mm}$ polyethylene converter placed over the thick silicon detector. Also shown in Figure 3 is the experimentally measured spectrum for the thick silicon detector without the converter layer reproduced from Figure 2 for the purposes of comparison.

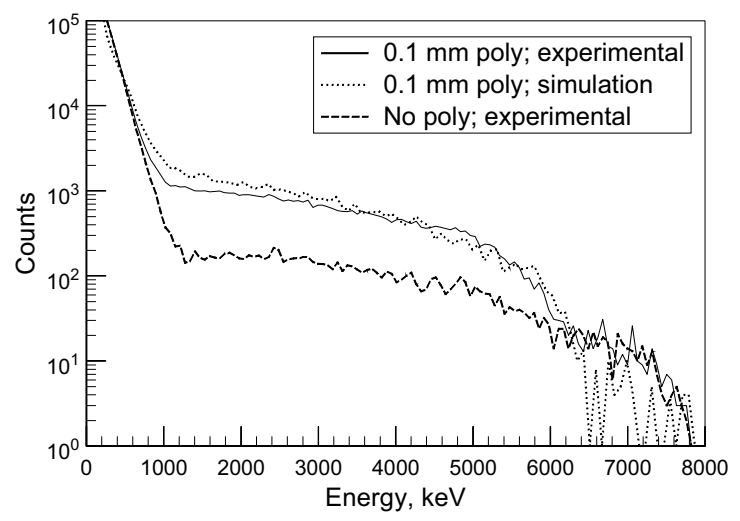

Fig. 3. Response of the thick silicon detector with a $0.1 \mathrm{~mm}$ thick polyethylene converter layer to the mixed radiation field. Spectra obtained from both experimental measurement and GEANT4 based simulations are shown. Also shown is the experimental response of the thick silicon detector without a converter layer for comparison purposes.

The low energy exponential tail previously attributed to Compton scattered gamma-rays and elastically scattered silicon recoils is evident. This part of the spectra is almost independent of the presence of the converter. The main difference in the spectra between the $0.1 \mathrm{~mm}$ polyethylene converted case and the bare case is the significantly increased number of events between approximately $1 \mathrm{MeV}$ and $6 \mathrm{MeV}$. These events correspond to the contribution of proton recoils associated with neutron interactions within the polyethylene converter. The edge in the spectra at about $6 \mathrm{MeV}$ is the energy deposited by protons with a $300 \mu \mathrm{m}$ range in silicon corresponding to the actual thickness of the detector. Protons with energy in excess of $6 \mathrm{MeV}$ were expected to pass through the finite thick detector without depositing all of their kinetic energy.

\section{B. SOI Microdosimeter Response}

Figure 4 shows the experimentally measured microdosimetric spectra obtained from the SOI microdosimeter with and without polyethylene converter layers.

In the case of the bare SOI microdosimeter the spectrum in the region of lineal energies between approximately 1 and 10 $\mathrm{keV} / \mu \mathrm{m}$ corresponds to secondary electrons produced mainly as a result of the interaction of the gamma component of the field within the detector. Extension of the spectrum to lower lineal energies as would be expected from theoretical considerations for electrons was not displayed on account of an energy cut made at about $1 \mathrm{keV} / \mu \mathrm{m}$ due to the presence of electronic noise in the original MCA spectrum. In the unnormalised spectra, not shown in this report, an equivalent number of events in both the bare detector and converter covered cases was observed for this region of lineal energies. This confirms that these events are associated with interactions of photons within the SV of the SOI microdosimeter and that it is possible to neglect any contribution attributable to the presence of the converter.

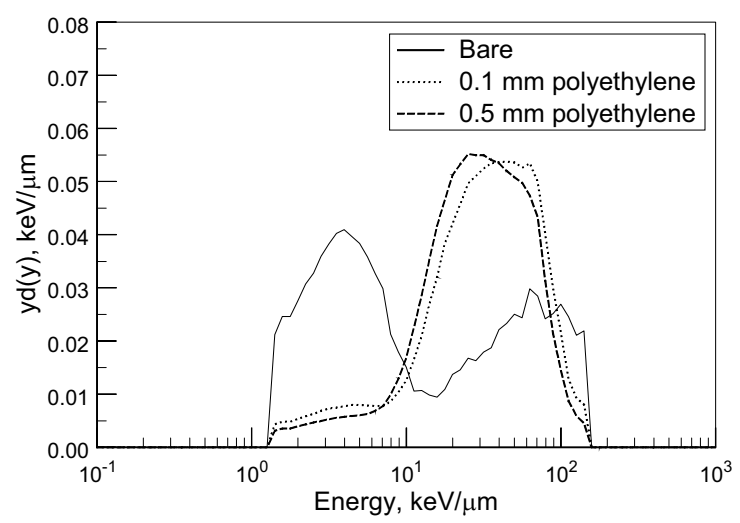

Fig. 4. Dose normalized experimentally measured microdosimetric spectrum obtained from the SOI microdosimeter in response to the mixed radiation field with and without the presence of a polyethylene converter layer.

In the bare SOI microdosimeter response for lineal energies between 10 and $200 \mathrm{keV} / \mu \mathrm{m}$ the contribution of elastic and inelastic reactions products resulting from direct interactions of neutrons with the silicon nuclei of the SOI microdosimeter can be observed. The significantly increased contribution in the region from 10 to $100 \mathrm{keV} / \mu \mathrm{m}$ in the polyethylene converter cases can be attributed to the recoil protons produced in the converter layer. The proton edge can be observed at a lineal energy of approximately $80 \mathrm{keV} / \mu \mathrm{m}$.

Comparing the spectra obtained between that of the $0.1 \mathrm{~mm}$ and $0.5 \mathrm{~mm}$ thick converter a shift to lower lineal energies was observed. This can be attributed to a relative increase in the number of protons with higher energy emerging from the thicker converter. An increase in proton energy is associated with a decrease in the lineal energy of the proton.

Figure 5 shows the experimentally measured microdosimetric spectra for the $0.5 \mathrm{~mm}$ converter case along 
with the GEANT4 simulation for this case. The gamma component of the radiation field was not included in the simulation. The contribution of lower lineal energy events is absent in the simulated spectrum as expected due to the absence of Compton electrons.

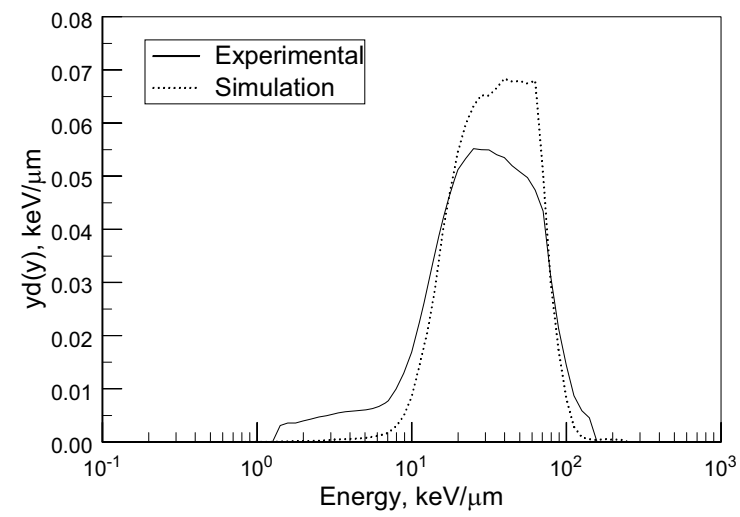

Fig. 5. Experimentally measured SOI microdosimeter response with a 0.5 mm thick polyethylene converter shown with the simulated SOI microdosimeter response. The gamma component of the radiation field was not included in the simulation.

Failure to include the gamma component results in a slightly different shape and amplitude in the dose normalised microdosimetric spectra in the region between 10 and 100 $\mathrm{keV} / \mu \mathrm{m}$. Some events with very high lineal energies were evident in the simulated response which were not evident in the experimental response. This was due to a lower upper energy threshold in the spectroscopy amplifier of the data acquisition system used in the experiment.

Figure 6 shows the contribution of crossers, stoppers and starters to the total microdosimetric spectrum of the simulated SOI microdosimeter response with a $0.5 \mathrm{~mm}$ polyethylene converter layer.

The maximum lineal energy of the stoppers was about 70 $\mathrm{keV} / \mu \mathrm{m}$ which corresponds quite closely to the proton edge. The shape of the distal edge of the stoppers corresponds to the variance in the chord length distribution for the rectangular shaped SV of the SOI microdosimeter. The low energy tail of the stoppers is due to recoil protons produced within the converter layer which have a range of less than $5 \mu \mathrm{m}$ in silicon.

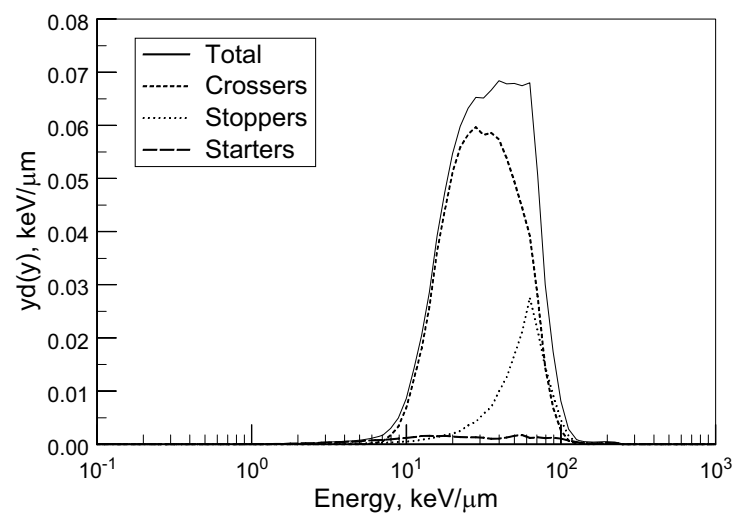

Fig. 6. GEANT4 simulated response of the SOI microdosimeter with a 0.5 $\mathrm{mm}$ polyethylene converter layer showing the contribution of crossers, stoppers and starters to the total microdosimetric spectrum. The gamma component of the radiation field was not included in the simulation.

\section{DISCUSSION}

For application of the SOI microdosimeter, or a related technology, in the field of radiation protection, consideration needs to be given to the detection efficiency. The measurements performed in this work were recorded with an acquisition time of about 12 hours, at a dose rate of approximately $10 \mu \mathrm{Gy}^{-1} \mathrm{~s}^{-1}$, and with a device sensitive area of approximately $3 \mathrm{~mm}^{2}$. Increasing the sensitive area to $3 \mathrm{~cm}^{2}$ and decreasing the event statistics by a factor of two would allow the counting time to be reduced to approximately 3 minutes for the same field intensity.

The converter thickness should be selected to reflect the microdosimetric spectra for a particular depth in soft tissue. For neutrons with energies up to $10 \mathrm{MeV}$ a reasonable approximation of particle equilibrium can be expected for a polyethylene converter thickness of $1 \mathrm{~mm}$.

For the same primary radiation spectra the secondaries spectra and particle type can vary considerably in different organs [10]. This will lead to a change in the microdosimetric spectra as was recently demonstrated [11]. For correct measurement of the equivalent dose with a SOI microdosimeter the converter composition should also match the organ of interest.

\section{CONCLUSION}

The application of a SOI microdosimeter in a low dose rate environment of mixed gamma and neutron radiation was demonstrated. GEANT4 simulations where found to be in good agreement with the experimental measurements suggesting that spectra of neutrons at the point of measurements and the model of the microdosimeter is satisfactory and can be used for future simulations. To cover the low dose range required in radiation protection the sensitive area of a personal microdosimeter should be 
increased. This presents a challenge in terms of the ability of the device to measure events with low lineal energies due to the increased noise associated with a larger device capacitance. The segmentation and multiplayer approach to a suitable SOI microdosimeters with a purpose designed ASIC has been proposed by CMRP and is under development. This will allow measurement of biological doses down to approximately $1 \mathrm{mSv}$ with an accuracy of $2-3 \%$.

\section{ACKNOWLEDGMENT}

We thank the staff of CSIRO Minerals for use of the neutron irradiation facility.

\section{REFERENCES}

[1] ICRU. Report N60, 1991

[2] H.H. Rossi and M. Zaider, "Microdosimetry and its Applications," Springer ISBN 3-540-58541-9, 1996.

[3] P.D. Bradley, A.B. Rosenfeld and M. Zaider, (invited paper), "Solid State Microdosimetry," Nucl.Instr.Meth. in Phys. Research B, vol. 184, pp. 135-157, 2001.

[4] I.M. Cornelius, A.B. Rosenfeld, R. Seigele, D.D. Cohen, "LET dependence of the charge collection efficiency of silicon microdosimeters," IEEE Trans. on Nucl. Sci, vol. 50, no. 6, pp. 23732379, 2003.

[5] P.D. Bradley and A.B. Rosenfeld, "Tissue Equivalence Correction for Silicon Microdosimetry Detectors in Boron Neutron Capture Therapy," Medical Physics, vol. 25, pp. 2220-2225, 1998.

[6] S. Agosteo, A. Castoldi, L. Castellani, P. Colautti, G.D. Angelo, L. De Nardo, A. Favalli, I. Lippi, R. Martinelli, G. Tornielli and P. Zotto, "A Feasibility Study of a single Event Spectrometer based on semiconductor Devices,” Radiat. Prot. Dosim., vol. 99, no. 1-4, pp. 343-346, 2002.

[7] J.D. Kinnison, R.H. Maurer, D.R. Roth and R.C. Haight, "High-Energy Neutron Spectroscopy with Thick Silicon Detectors," Rad. Res., vol. 159, pp. 154-160, 2003.

[8] I. Cornelius and A. Rosenfeld, "Monte Carlo verification in Fast Neutron Therapy," IEEE Trans. on Nucl. Sci, vol. 51, no. 3, 873-877, 2004.

[9] IAEA, "Compendium of Neutron Spectra and Detector Responses for Radiation Protection Purposes," Technical Report Series, no. 403, (2001).

[10] A.B. Rosenfeld, A.J. Wroe, I.M. Cornelius, M. Reinhard and D. Alexiev, "Analysis of Inelastic Interactions for Therapeutic Proton Beams using Monte Carlo simulation," IEEE Trans. on Nucl. Sci. vol. 51, no. 6, pp. 3019-3025, 2004.

[11] A. Wroe, I. Cornelius, A. Rosenfeld, V. Pisacane, J.F. Ziegler, M.E. Nelson, F. Cucinotta, M. Zaider and J. Dicello, "Microdosimetric simulations within heterogenius structures," IEEE Trans. on Nucl. Sci., vol. 52 , no. 6 (to be published) 